\title{
BOURSES DE RECHERCHE DE L'ACMU 2007
}

\section{Le Comité de recherche de l'ACMU est fier de vous présenter le Concours de bourses de recherche pour l'année 2007.}

\section{Cette année, la présentation des demandes se fera EN LIGNE seulement au : www.pretx.org/CAEP-SUBMIT/}

La date limite pour faire une demande de bourse en ligne est vendredi, le 15 décembre 2006 à 12 h (midi) (HNE). Les résultats du concours seront envoyés au chercheur principal jeudi le 15 mars 2007.

Les demandes provenant de divers milieux de pratique de la médecine d'urgence seront considérées, soit les milieux universitaire, non universitaire, communautaire et rural. On encourage plus particulièrement la participation des résidents et des fellows. L'appui financier maximal pour un projet individuel sera de 5000 . La bourse ne peut PAS être considérée comme un supplément à d'autres sources de financement. Le chercheur principal et superviseur attitré (pour les demandes des résidents et des fellows) doit être membre de l'ACMU au moment de l'attribution des fonds. Aucun financement ne sera accordé pour l'inscription à des congrès, les frais de déplacement ou l'achat d'équipement.

\section{Conditions préalables pour soumettre un projet :}

- Le chercheur principal doit être membre de l'ACMU au moment de la demande.

- Le chercheur principal doit avoir fait moins de huit ans de formation postdoctorale (École de médecine).

- Pour les résidents et les fellows qui sont chercheurs principaux, le superviseur attitré doit joindre une lettre d'accompagnement au projet.

\section{Processus de demande :}

Les projets ne doivent pas dépasser cinq pages à simple interligne, en excluant la bibliographie et les annexes. Une police de taille 12 et des marges non ajustées sont obligatoires. Il n'est pas nécessaire de joindre une lettre de présentation. Le projet doit consister en un dossier WORD comportant les titres et les numéros de pages qui suivent :

1. Résumé structuré du projet de recherche (page 1).

2. Introduction, Exposé raisonné, Hypothèse de recherche et Méthodes (limite : 3 pages; pages 2-4).

3. Échéancier, Impact de l'étude proposée, Plans ultérieurs (limite : 1 page; page 5).

4. Bibliographie (Annexe A, limite de 30 références et de 2 pages).

5. Outil de collecte des données pour la recherche (Annexe B; si plus d'un outil, indiquer B1, B2).

6. Budget détaillé et justification (Annexe C).

7. Curriculum vitae du chercheur principal SEULEMENT (Annexe D).

8. Lettre d'accompagnement du superviseur attitré (voir le dernier point à «conditions préalables») (Annexe E; 1 page).

\section{Les évaluateurs se pencheront principalement sur :}

- la pertinence du projet pour la médecine d'urgence;

- la qualité méthodologique;

- l'originalité du sujet et de la méthodologie;

- le niveau de formation; et

- la capacité à compléter l'étude proposée.

Les chercheurs qui auront été choisis devront fournir une lettre indiquant qu'ils vont :

- remettre un rapport final sur le projet de recherche au plus tard 15 mois après l'attribution des fonds;

- retourner toutes sommes non utilisées à l'ACMU au plus tard 15 mois après l'attribution des fonds; et

- présenter les résultats de l'étude lors de l'Assemblée annuelle de l'ACMU.

Pour de plus amples renseignements, veuilles communiquer avec le $\mathrm{D}^{\mathrm{r}}$ Ran Goldman, Bourses de recherche de l'ACMU 2006, Siège social de l'ACMU, 1785, prom. Alta Vista, bureau 104, Ottawa ON K1G 3Y6; 613 523-3343 ou 800 463-1158 poste 17, fax 613 523-0190, committees@ caep.ca 